Pacific Journal of Mathematics

FREE LATTICE-ORDERED MODULES 


\section{FREE LATTICE-ORDERED MODULES}

\section{A. BIGARD}

The aim of this paper is to show that the theory of free lattice-ordered groups developed by E. C. Weinberg in the abelian case can be generalized to modules over a totally ordered Ore domain $A$. The main result is that for every torsion-free ordered $A$-module $M$, there exists a free $f$-module over $M$. The generalization given will be seen to be, in a certain sense, the best possible.

All rings and modules considered will be assumed to be unital. Let $A$ be a partially ordered ring and $A_{+}$its order. If $M$ is a left $A$-module, we say that $P \subseteq M$ is an order on $M$ if:

$P+P \subseteq P, A_{+} P \subseteq P$, and $P \cap-P=\{0\}$. If such a $P$ is given, we say that $M$ is a partially ordered (or ordered) module. If $P$ is a total order on $M$, that is, if $M=P \cup-P$, we say that $M$ is a totally ordered module. Let $M$ and $N$ be partially ordered $A$-modules and let $f$ be a mapping from $M$ to $N$. Then $f$ is an $o$-homomorphism if $f$ is a monotonic homomorphism of $A$-modules. The $o$-homomorphism $f$ is an $o$-isomorphism if $f$ is one-to-one and if $f^{-1}$ is an o-homomorphism.

1. Some properties of $f$-modules. In this section, $A$ will denote a directed p.o. ring. An $A$-module $M$ which is lattice-ordered by the order $P$ is called a lattice-ordered module or $l$-module. Products of lattice-ordered modules are defined in a natural way. If $M$ and $N$ are $l$-modules, an homomorphism $f$ from $M$ to $N$ is called a $l$-homomorphism if, for $x, y \in M$ :

$$
f(x \vee y)=f(x) \vee f(y) \text { and } f(x \wedge y)=f(x) \wedge f(y) .
$$

An $f$-module $M$ is a lattice-ordered module which is a subdirect product of totally ordered modules. This definition was first introduced in [1] and [3].

Recall that a convex $l$-subgroup $S$ in a commutative l.o. group $G$ is called prime if $G / S$ is totally ordered. The following theorem gives useful characterizations of $f$-modules.

THEOREM 1. Let $M$ be a lattice-ordered module over a unital directed ring $A$. The following are equivalent:

(1) $M$ is an $f$-module.

(2) For $x, y \in M$ and $0 \leqq \lambda \in A, \lambda(x \vee y)=\lambda x \vee \lambda y$ and $\lambda(x \wedge y)=$ $\lambda x \wedge \lambda y$. 
(3) $x \wedge y=0$ implies $\lambda x \wedge y=0$ for all $0 \leqq \lambda \in A$.

(4) Every minimal prime subgroup of $M$ is a submodule.

Proof. (1) implies (2): This is clear since (2) is satisfied in a totally ordered module.

(2) implies (3): If $x \wedge y=0$, then we have:

$$
0 \leqq \lambda x \wedge y \leqq(\lambda \vee I) x \wedge(\lambda \vee I) y=(\lambda \vee I)(x \wedge y)=0
$$

(3) implies (4): Let $S$ be a minimal prime subgroup. Then, $x \in S$ if and only if there exists $y \notin S$ with $x \wedge y=0$. [2]. Thus, if $x \in S$ and $0 \leqq \lambda \in A$, we have $\lambda x \in S$. Since $A$ is directed, $S$ is a submodule.

(4) implies (1): Let $\left(S_{i}\right)_{i \in I}$ be the family of all minimal prime subgroups of $M$. Then each quotient $M / S_{i}$ is a totally ordered module and $M$ is a subdirect product of these modules.

If $A$ is not unital, then (1), (3), and (4) are equivalent but condition (2) is weaker (see [3]).

In the sequal, we shall be concerned mainly with torsion-free modules, that is modules in which $\lambda x=0$ implies $\lambda=0$ or $x=0$. The following property is useful:

Proposition 1. If $A$ is totally ordered, every torsion-free $f$ module $F$ is a subdirect product of torsion-free totally ordered modules.

Let $S$ be a minimal prime subgroup of $F$. Suppose that $\lambda \neq 0$ and $\lambda x \in S$. We may assume $\lambda>0$, as $A$ is totally ordered. As in the proof of Theorem 1, there exists $y \notin S$ with $\lambda x \wedge y=0$. This implies $\lambda(x \wedge y)=\lambda x \wedge \lambda y=0$, and hence $x \wedge y=0$. As $y \notin S$, we obtain $x \in S$. This proves that $M / S$ is torsion-free and the theorem follows.

As in the theory of ordered groups, $P$ is an isolated order on $M$ if $\lambda>0$ and $\lambda x \in P$ implies $x \in P$.

Proposition 2. Every torsion-free f-module is isolated.

Proof. If $\lambda>0$ and $\lambda x \geqq 0$, we have $\lambda(-x \vee 0)=-\lambda x \vee 0=0$, hence $-x \vee 0=0$ and $x \geqq 0$.

Conversely, it is clear that when $A$ is totally ordered, every isolated module is torsion-free.

2. Embedding an order in a total order. In this section, we consider only torsion-free modules over a totally ordered unital ring $A$. This is not as restrictive as it seems, since the existence of a 
nontrivial torsion-free module implies that $A$ has no zero divisors, and an $f$-ring with no zero divisors is totally ordered.

Lemma 1. Let $M$ be a torsion-free A-module. For every $x \in M$, $A_{+} x$ is an order.

Proof. Suppose that $y \in A_{+} x \cap-A_{+} x$, so that $y=\lambda x=-\mu x$. The relation $(\lambda+\mu) x=0$ implies $\lambda+\mu=0$ or $x=0$. In the first case, $\lambda=-\mu \in A_{+} \cap-A_{+}$so in each case $y=0$.

Lemma 2. Let $P$ and $Q$ be two orders on $M$. Then $P-Q$ is an order if and only if $P \cap Q=0$.

Proof. The condition is necessary, since $P \cap Q \subseteq(P-Q) \cap(Q-P)$. For the converse, suppose $P \cap Q=0$ and let $y \in(P-Q) \cap(Q-P)$. Then $y=p-q=q^{\prime}-p^{\prime}$, and $p+p^{\prime}=q+q^{\prime} \in P \cap Q=0$. Hence, $p=-p^{\prime} \in P \cap-P=0, q=-q^{\prime} \in Q \cap-Q=0$, and it follows that $y=0$.

The ring $A$ is said to be a left Ore domain if $A$ admits a left quotient field. Equivalently, $A$ has no zero divisors and satisfies the following condition:

( I ) If $\rho \neq 0$ and $\sigma \neq 0, A \rho \cap A \sigma \neq 0$.

Clearly, when $A$ is totally ordered, this condition can be replaced by the following:

(II) If $0<\rho$ and $0<\sigma, A_{+} \rho \cap A_{+} \sigma \neq 0$.

Theorem 2. Let $A$ be a totally ordered ring with no divisors of zero. The following are equivalent:

(1) $A$ is a left Ore domain.

(2) In a torsion-free A-module, every order is contained in a total order.

(3) In a torsion-free A-module, every order is contained in an isolated order.

Proof. (1) implies (2): By Zorn's lemma, every order is contained in a maximal order. It remains to show that each maximal order $P$ is total. If not, suppose $b \notin P \cup-P$. As $P \subset P+A_{+} b$ (strictly), $P+A_{+} b$ fails to be an order. By Lemma 2, $P \cap-A_{+} b \neq 0$ and there exists $\rho>0$ with $\rho b \in-P$. Similarly, $P-A_{+} b$ is not an order, $P \cap A_{+} b \neq 0$, and there exists $\sigma>0$ with $\sigma b \in P$. By condition (II), there exists $\lambda>0$ and $\mu>0$ with $\lambda \rho=\mu \sigma>0$. Hence $\lambda \rho b=\mu \sigma b \in P \cap-P=0$. This implies $b=0$, which is a contradiction. Hence $P$ is a total order.

(2) implies (3): This is clear from Proposition 2. 
(3) implies (1): Consider $A$ as a left-module on itself. Take $0<\rho$ and $0<\sigma$. If $A_{+} \rho \cap A_{+} \sigma=0, A_{+} \rho-A_{+} \sigma$ is an order by Lemma 2. Hence it is contained in an isolated order $P$, and thus $\rho 1 \in P$ and $\sigma(-1) \in P$. Then $1 \in P$ and $-1 \in P$, which is a contradiction.

COROLlaRY 1. Let $A$ be a totally ordered left Ore domain. Let $f$ be an o-homomorphism of the torsion-free module $M$ ordered by $P$ into a torsion-free totally ordered module $T$. There exists a total order $P_{0}$ which contains $P$ such that $f(x)>0$ implies $x \in P_{0}$.

To see that $S=\{x \mid f(x)>0\} \cup\{0\}$ is an order on $M$, note that $S+S \subseteq S$ and $S \cap-S=\{0\}$. Also for $\lambda>0$ and $0 \neq x \in S, f(x)>0$ and hence $f(\lambda x)=\lambda f(x)>0$ since $T$ is torsion-free. As $P \cap-S=0$, $P+S$ is an order by Lemma 2. The corollary then follows from Theorem 1.

Corollary 2. Let $A$ be a totally ordered left Ore domain and let $M$ be a torsion-free $A$-module ordered by $P$. The intersection of all total orders containing $P$ is the set $\bar{P}$ of elements $x \in M$ for which there exists $\lambda>0$ with $\lambda x \in P$.

Each total order containing $P$ is isolated and hence contains $\bar{P}$. Suppose $x \notin \bar{P}$, so that $P \cap A_{+} x=0$. By Lemma 2, $P-A_{+} x$ is an order. By Theorem 2, $P-A_{+} x$ is contained in a total order $Q$. Since $-x \in Q$ and $x \neq 0, x \notin Q$.

Theorem 3. Let $A$ be a totally ordered left Ore domain. If $M$ is an $A$-module ordered by $P$, these are equivalent:

(1) $P$ is isolated.

(2) $M$ is torsion-free and $P$ is an intersection of total orders.

(3) $M$ can be embedded in a direct product of totally ordered torsion-free modules.

(4) $M$ can be embedded in a torsion-free f-module.

Proof. (1) implies (2): This follows directly from Corollary 2, as $P=\bar{P}$.

(2) implies (3): Let $\left(P_{i}\right)_{i \in I}$ be the set of all total orders containing $P$. If we denote by $M_{i}$ the module $M$ ordered by $P_{i}$, there is a canonical embedding of $M$ into the direct product of the modules $M_{i}$.

(3) implies (4): Clear.

(4) implies (1): This follows from Proposition 1. 
3. Free $f$-modules. Let $A$ be a totally ordered left Ore domain, and let $M$ be a torsion-free $A$-module ordered by $P$. A torsion-free $f$-module $L$ will be called free over $M$ if:

(1) There exists an injective o-homomorphism $\varphi$ from $M$ to $L$.

(2) For every torsion-free $f$-module $F$ and every $o$-homomorphism $f$ from $M$ to $F$, there exists a unique $l$-homomorphism $\bar{f}$ from $L$ to $F$ such that $\bar{f} \circ \phi=f$.

It is not difficult to show that $L$ is determined up to an $l$ isomorphism. To show that such an $L$ exists, we use the two following lemmas:

LEMma 3. If $x_{\alpha \beta}(\alpha \in R, \beta \in S)$ and $x_{\gamma \delta}(\gamma \in U, \delta \in V)$ are two finite families of elements in a lattice-ordered module,

$$
\bigvee_{\alpha \in R} \bigwedge_{\beta \in S} x_{\alpha \beta}-\bigvee_{\gamma \in U} \bigwedge_{\delta \in V} x_{\gamma \delta}=\underbrace{}_{(\alpha, \sigma) \in R \times(V S \times U)} \bigwedge_{(\beta, \gamma) \in S \times U}\left(x_{\alpha \beta}-x_{(\gamma)(\sigma(\beta, \gamma))}\right) .
$$

Proof.

$$
\begin{aligned}
& \bigvee_{R} \bigwedge_{S} x_{\alpha \beta}-\bigvee_{U} \bigwedge_{V} x_{\gamma \delta}=\bigvee_{R} \bigwedge_{S} \bigwedge_{U} \underset{V}{\mathbf{V}}\left(x_{\alpha \beta}-x_{\gamma \delta}\right)=\bigvee_{R} \bigwedge_{S \times U} \underset{V}{\mathbf{V}}\left(x_{\alpha \beta}-x_{\gamma \delta}\right) \\
& =\bigvee_{R} \underset{\rho \in(V S \times U)}{\bigvee_{S \times U}} \bigwedge_{S \times \beta}\left(x_{\alpha \beta}-x_{(r)(\sigma(\beta, \gamma))}\right) \\
& =\bigvee_{(\alpha, \sigma) \in R \times(V S \times U)} \bigwedge_{(\beta, \gamma) \in S \times U}\left(x_{\alpha \beta}-x_{(\gamma)(\sigma(\beta, \gamma))}\right) \text {. }
\end{aligned}
$$

Lemma 4. Let $N$ be a f-module and $K$ a submodule of $N$. The $f$-submodule generated by $K$ is the set $K^{\prime}$ of all elements $\mathbf{V}_{\alpha \in R} \Lambda_{\beta \in S} x_{\alpha \beta}$ with $x_{\alpha \beta} \in K$.

Proof. By Lemma $3, K^{\prime}$ is an $l$-subgroup of $N$. If $\lambda \geqq 0$, it follows from Theorem 1 that: $\lambda \mathbf{V}_{R} \Lambda_{S} x_{\alpha \beta}=\mathbf{V}_{R} \Lambda_{S} \lambda x_{\alpha \beta}$. Since the ring is assumed to be directed, $K^{\prime}$ is a submodule.

THEOREM 4. Let $\left(P_{i}\right)_{i \in I}$ be the set of all total orders on $M$ containing $P$, and denote by $M_{i}$ the module $M$ ordered by $P_{i}$. Let $\varphi$ be the canonical map of $M$ into $\prod_{i \in I} M_{i}$. Then the f-submodule $L$ of $\prod_{i \in I} M_{i}$ generated by $\varphi(M)$ is free over $M$.

Proof. Suppose $f$ is an o-homomorphism from $M$ into a torsionfree $f$-module $F$. If $x \in L$, then by Lemma $4, x=\bigvee_{R} \Lambda_{S} \varphi\left(x_{\alpha \beta}\right)$ where $x_{\alpha \beta} \in M$.

Let $\bar{f}(x)=\mathrm{V}_{R} \Lambda_{S} f\left(x_{\alpha \beta}\right)$. To show that $\bar{f}$ is a mapping, it is sufficient to show, by Lemma 3 , that $\mathbf{V}_{R} \Lambda_{s} \varphi\left(x_{\alpha \beta}\right)=0$ implies $\mathrm{V}_{R} \Lambda_{S} f\left(x_{\alpha \beta}\right)=0$.

By Proposition 1, we may assume that $F$ is totally ordered. By 
Corollary 1 of Theorem 2 , there exists a total order $P_{0}$ containing $P$ such that $f(x)>0$ implies $x \in P_{0}$.

If $\mathbf{V}_{R} \Lambda_{s} f\left(x_{\alpha \beta}\right)>0$, there exists $\alpha \in R$ such that for each $\beta \in S$, $f\left(x_{\alpha \beta}\right)>0$, which implies $x_{\alpha \beta} \in P_{0}$. It follows that $V_{R} \Lambda_{s} x_{\alpha \beta}>0$ (modulo $P_{0}$ ) and $\mathbf{V}_{R} \Lambda_{S} \varphi\left(x_{\alpha \beta}\right) \neq 0$. Alternatively, if $\mathbf{V}_{R} \Lambda_{s} f\left(x_{\alpha \beta}\right)<0$, there exists for each $\alpha \in R, a \beta \in S$ such that $f\left(x_{\alpha \beta}\right)<0$. Thus $x_{\alpha \beta} \in$ $-P_{0}$ and it follows that $\mathrm{V}_{R} \Lambda_{S} x_{\alpha \beta}<0$ (with respect to $P_{0}$ ). Hence $\mathbf{V}_{R} \Lambda_{S} \varphi\left(x_{\alpha \beta}\right) \neq 0$. Now, it is clear that $\bar{f}$ is a mapping. By Lemma $3, \bar{f}$ is a group homomorphism. The theorem follows easily.

CoRollary. Let $A$ be a totally ordered ring with no divisors of zero. The following are equivalent:

(1) A is a left Ore domain.

(2) For every torsion-free ordered module $M$, there exists a free $f$-module over $M$.

Proof. By Theorem 4, (1) implies (2). Conversely, if $\varphi$ is the $o$-homomorphism of $M$ into the free $f$-module $L$ over $M$, the positive cone of $M$ is a subset of $Q=\{x \mid \varphi(x) \geqq 0\}$, which is an isolated order. Thus, (2) implies (1) by Theorem 2.

Note that $\varphi$ is an 0 -isomorphism of $M$ into $L$ if and only if $M$ is isolated.

It is now easy to construct the free $f$-module over an arbitrary set $E$. Let $M$ be the free module generated by $E$, and trivially order $M$ by $P=\{0\}$. The free $f$-module $L$ generated by $M$ is a free $f$-module over $E$, with obvious definitions.

\section{REFERENCES}

1. A. Bigard, Contribution à la théorie des groupes réticulés, Thèse Sc. Math., Paris, 1969.

2. P. Conrad, Introduction à la théorie des groupes réticulés, Secrétariat mathématique, Paris, 1967.

2. S. A. Steinberg, Lattice-ordered rings and modules, Ph. D. Thesis, Urbana, Illinois, 1970.

4. E. C. Weinberg, Free lattice-ordered abelian groups, Math. Annalen t, 151 (1963), 187-199 and t. 159 (1965), 217-222.

Received July 19, 1972 and in revised form May 4, 1973.

Centre Universitaire du Mans 


\section{PACIFIC JOURNAL OF MATHEMATICS}

\section{EDITORS}

RICHARD ARENS (Managing Editor) University of California

Los Angeles, California 90024

R. A. Beaumon'T

University of Washington Seattle, Washington 98105
J. Dugundj1*

Department of Mathematics University of Southern California Los Angeles, California 90007

D. Gilbarg and J. Milgram Stanford University Stanford, California 94305

\section{ASSOCIATE EDITORS}

E. F. BECKENBACH

B. H. NeUMANN

F. WOLF

K. YOSHIDA

\section{SUPPORTING INSTITUTIONS}

UNIVERSITY OF BRITISH COLUMBIA CALIFORNIA INSTITUTE OF TECHNOLOGY UNIVERSITY OF CALIFORNIA MONTANA STATE UNIVERSITY UNIVERSITY OF NEVADA NEW MEXICO STATE UNIVERSITY OREGON STATE UNIVERSITY UNIVERSITY OF OREGON OSAKA UNIVERSITY

\section{UNIVERSITY OF SOUTHERN CALIFORNIA STANFORD UNIVERSITY UNIVERSITY OF TOKYO UNIVERSITY OF UTAH WASHINGTON STATE UNIVERSITY UNIVERSITY OF WASHINGTON AMERICAN MATHEMATICAL SOCIETY NAVAL WEAPONS CENTER}

* C. R. DePrima California Institute of Technology, Pasadena, CA 91109, will replace J. Dugundji until August 1974. 


\section{Pacific Journal of Mathematics}

\section{Vol. 49, No. $1 \quad$ May, 1973}

A. Bigard, Free lattice-ordered modules ...........................

Richard Bolstein and Warren R. Wogen, Subnormal operators in strictly cyclic

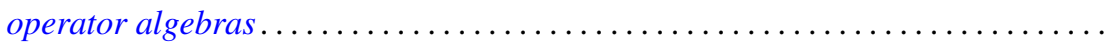

Herbert Busemann and Donald E. Glassco, II, Irreducible sums of simple

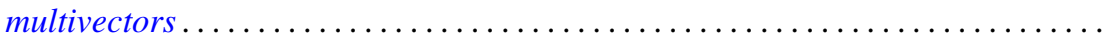

W. Wistar (William) Comfort and Victor Harold Saks, Countably compact groups

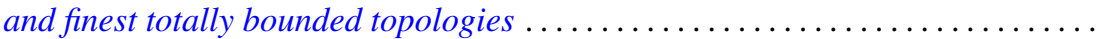

Mary Rodriguez Embry, Maximal invariant subspaces of strictly cyclic operator

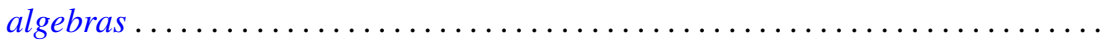

Ralph S. Freese and James Bryant Nation, Congruence lattices of semilattices......

Ervin Fried and George Grätzer, A nonassociative extension of the class of

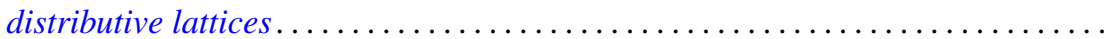

John R. Giles and Donald Otto Koehler, On numerical ranges of elements of locally

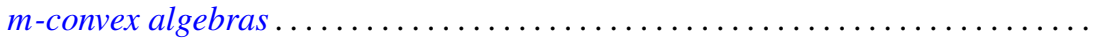

David A. Hill, On dominant and codominant dimension of $\mathrm{QF}-3$ rings ........ John Sollion Hsia and Robert Paul Johnson, Round and Pfister forms over $R(t) \ldots$ I. Martin (Irving) Isaacs, Equally partitioned groups . . . . . . . . . . . . . .

Athanassios G. Kartsatos and Edward Barry Saff, Hyperpolynomial approximation of solutions of nonlinear integro-differential equations.

Shin'ichi Kinoshita, On elementary ideals of $\theta$-curves in the 3-sphere and 2-links in the 4-sphere

Ronald Brian Kirk, Convergence of Baire measures

R. J. Knill, The Seifert and Van Kampen theorem via regular covering spaces ..

Amos A. Kovacs, Homomorphisms of matrix rings into matrix rings ..

Young K. Kwon, HD-minimal but no $H D$-minimal ..........

Makoto Maejima, On the renewal function when some of the mean renewal lifetimes are infinite

Juan José Martínez, Cohomological dimension of discrete modules over profinite groups.

W. K. Nicholson, Semiperfect rings with abelian group of units

Louis Jackson Ratliff, Jr., Three theorems on imbedded prime divisors of principal ideals.

Billy E. Rhoades and Albert Wilansky, Some commutants in $B(c)$ which are almost matrices

John Philip Riley Jr., Cross-sections of decompositions . . .

Keith Duncan Stroyan, A characterization of the Mackey uniformity $m\left(L^{\infty}, L^{1}\right)$ for

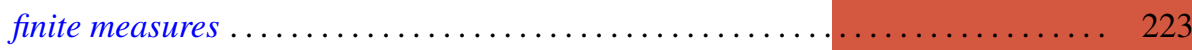

Edward G. Thurber, The Scholz-Brauer problem on addition chains . . . . . . . . . 229

Joze Vrabec, Submanifolds of acyclic 3-manifolds ............

Philip William Walker, Adjoint boundary value problems for compactified singular

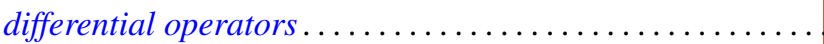

